\section{CÓMO ABORDAR LA HIPERACTIVIDAD EN EL ÁMBITO ESCOLAR}

HOW TO APPROACH HYPERACTIVE CHILDREN AT SCHOOL

María Reina Mejía ${ }^{1}$

Isolisbeth González**

Ciro R. Cohen***.

UPEL-IPB

Recibido: 25-05-07

\section{RESUMEN}

El presente artículo de naturaleza documental analiza algunas estrategias pedagógicas para aborda la hiperactividad en infantes en el ámbito escolar. A través de la revisión crítica del referencial teórico, se describen las situaciones de aprendizaje que el docente puede reconocer en un niño hiperactivo. Se establece que el niño hiperactivo requiere de un docente mediador del aprendizaje, conductor de procesos de socialización e interacción grupal, de formación de valores y hábitos necesarios para conformar su personalidad y, lograr así, avances satisfactorios que le garanticen su permanencia dentro del sistema educativo. Asimismo, el docente debe apoyarse en un equipo multiprofesional que ofrezcan diversos servicios: prevención, detección, tratamiento y seguimiento del niño hiperactivo en el contexto escolar. Así, se concluye que las estrategias presentadas pueden ayudar al docente del Aula Regular Integrada a reforzar, dinamizar, afianzar y mediar el aprendizaje de manera organizada, planificada, correcta, específica e inclusiva. Descriptores: niño hiperactivo, rol del docente, estrategias pedagógicas.
Aceptado: 29-11-07

\section{ABSTRACT}

The following documentary paper is an analysis of some pedagogical strategies to cope with hyperactive children. Through the theory, learning situations to instruct children under this condition are described. It is observed that hyperactive children need teachers who effectively facilitate the learning process and promote socialization, peer work, moral values and habits; in other words, someone who can help them build their personality and guarantee their place within the school system. To achieve these goals, the teacher must count on a multiprofessional team working in areas such as prevention, detection, treatment and inspection of hyperactive children's performance at school. It is concluded that the analyzed strategies help teachers reinforce, establish and facilitate learning in an organized, planned, specific, inclusive and appropariate way.

Keywords: hyperactive children, teacher's role, pedagogical strategies

${ }^{1}$ Doctora en Ciencias de la Educación. Magíster en Orientación. Licenciada en Educación Mención Dificultades

del Aprendizaje. Docente Titular, Dedicación Exclusiva en la UPEL-IPB Programa de Preescolar. ** Doctora en Ciencias de la Educación. Magíster en Planificación Curricular. Profesora en la

Educación Preescolar. Docente a Tiempo Completo de la UPEL-IPB Programa De Preescolar. de Ciencias Sociales Mención Historia. Docente Titular Dedicación Exclusiva UPEL-IPB Programa de Preescolar. Coordinador del Núcleo de Investigación de Educación Especial (NIEDE).

El presente artículo se constituye en una revisión teórica de los postulados fundamentales que explican la atención del niño y niña con conductas hiperactivas en el contexto escolar. De allí que se aborda el rol de la escuela como ámbito donde convergen actores claves de integración que son los docentes, padres, familia y comunidad como agentes conductores de aprendizaje.

En tal sentido, se asumen autores como Uriarte (1989) que destacan la escuela como formadora de la infancia, la cual es confrontada con los lineamientos del Ministerio de Educación a través de la Resolución 2005 que establece puntos a seguir para apoyar la integración de alumnos con Necesidades Especiales al Aula Regular. Así mismo se consideran Autores tales como Fried y Bursuck (1999); Orella, J. (1991); Valet, R. (1991); Velasco, R. (1992); Castro, P. (1984); Gallardo, B. (1991); Mejía, M. (1999); Rief, S. (1999); Much (1996); Joselevich, E. (2003); Arcos, T. y Fernández, A. (2004); Asociación Americana de siquiatría (DSM-IV-TR) (2005); De La Garza, F. (2005) y Scandar, R. (2006) quienes desde puntos de vista coincidente plantean postulados que enfatizan el aprendizaje en el aula de la diversidad, el rol del docente en la atención de conductas hiperactivas y organización del ambiente escolar. Igualmente, se refieren estrategias pedagógicas para atender la hiperactividad y el rol del Equipo Multiprofesional para facilitar procesos eficaces en la integración, en consideración de los niveles y funciones que deben ser mediados junto al docente. De este modo se describen seguidamente los postulados teóricos que guían la concepción de la integración de educandos con necesidades especiales al aula regular.

En la actualidad, la dinámica de cambios que vivencia la Educación Venezolana impulsa una Didáctica Pedagógica que debe centrar sus acciones en promover experiencias de aprendizaje que atiendan las características individuales de los alumnos dentro de un ambiente enriquecedor. De este modo, los Centros Educativos abren sus espacios para la formación de niños y niñas con necesidades, intereses y potencialidades diferentes, permitiendo potenciar una diversidad de individuos en las aulas. Es así como se incorporan a los niños y niñas con necesidades especiales al aula regular con la intencionalidad de favorecer experiencias que contribuyan al pleno desarrollo de su personalidad.

Dentro de las necesidades especiales es frecuente encontrar conductas hiperactivas que deben ser atendidas con estrategias pedagógicas diversas que respondan a sus características particulares a fin de favorecer su integración con el grupo de niños del aula 
regular. Sin embargo, para comprender las implicaciones de la hiperactividad en niños y niñas con necesidades especiales es fundamental considerar los postulados teóricos que explican su deber ser para su atención plena en el contexto escolar. De allí que debemos destacar que la escuela es el ámbito donde convergen diversos niños (as), docentes, padres y comunidad, como agentes conductores del proceso de aprendizaje y de socialización de los niños (as) signados por antecedentes familiares, genéticos y físicos que interfieren y chocan con las medidas disciplinarias existentes en el recinto escolar.

Este grupo de personas responsables del proceso de aprendizaje deben entender que la escuela es el sitio apropiado para aprender a comunicarse, a expresar conductas, comportamientos, establecer relaciones sociales, compartir intereses, inquietudes, necesidades, tristezas y alegrías, recibir y compartir conocimientos, tal como lo plantea Uriarte (1989) quien señala a la escuela como la institución formadora más importante para la infancia, exige atributos al alumno que el hiperactivo no reúne, uno de los principales es la colaboración alumnos-maestros. También se requiere de disciplina y obediencia, tolerancia a la frustración, permanencia en el asiento trabajando, mantener fija la atención, realizar con precaución y paciencia las actividades, requisitos éstos fundamentales. Esta postura valoriza la acción de la escuela, en cuanto a las exigencias relacionadas con las normas de convivencia, cooperación y disciplina que los niños deben cumplir en el aula de la integración, y presenta la situación de desventaja del niño (a) hiperactivo (a) ante dichas normas así como su incapacidad para cumplirlas y lograr su aprendizaje acorde con las exigencias personales y grupales.

En tal sentido, el Ministerio de Educación, a través de la Resolución 2005, en su Artículo 7 establece que los planteles educativos y sus servicios de apoyo, responsables del proceso de integración escolar de los alumnos con necesidades especiales, coordinarán las actividades informativas, formativas, socio-culturales, deportivas, dirigidas a los padres y comunidad en general a fin de proporcionar la integración familiar y social del educando.

Por otra parte, el Ministerio de Educación (1.996) en las líneas políticas educativas de la modalidad de Educación Especial, establece que esta modalidad da apoyo al alumno integrándolo a la escuela regular y con los docentes que participan en el proceso; facilita estrategias de integración, organización escolar, adaptación curricular, equipos y materiales, metodologías y recursos, a través de una acción cooperativa entre especialistas y personal docente. Estos mandatos desinhiben paso a paso el deber ser del proceso de integración de los niños (as) con necesidades especiales al aula regular, señalando como responsables del proceso a padres, docentes y especialistas de educación, estos últimos son los responsables de brindar una gama de factores que visualizan el proceso de cooperación entre los diferentes participantes, que hacen vida activa en el aula de clase para llevar un proceso de aprendizaje acorde con las necesidades e intereses de los niños (as) y con las exigencias curriculares, para facilitar la progresiva integración de los sujetos con necesidades especiales del aula regular y por ende de la escuela en general.

En otro orden de ideas, la Ley para las Personas con Discapacidad, según Gaceta Oficial $N^{\circ} 38598$ de fecha 5 de Enero de 2007 en su Artículo $1^{\circ}$ sostiene que: "Las disposiciones de la presente ley tiene por objeto: "Regular los medios y mecanismos, que garanticen el desarrollo integral de las personas con discapacidad de manera plena y autónoma, de acuerdo con sus capacidades, y lograr la integración a la vida familiar y comunitaria”. (p. 1). Como podemos observar, dicho Artículo de la ley en referencia, legaliza y legitima el desarrollo integral de los sujetos con discapacidad tomando en cuenta sus potencialidades y aptitudes, y su integración a la sociedad.

Ante tal situación y para dar un poco de direccionalidad al proceso de integración del niño (a) con necesidades especiales al aula regular, es oportuno señalar los aspectos que reseñan Fried y Bursuck (1999) en cuanto a: organización física, rutinas para el trabajo del aula, clima del aula, reglas del aula y empleo del tiempo. Estos procesos implican: (a) la organización física incluye el ambiente del aula, el uso de espacios, paredes, iluminación, el piso, lugares para guardar materiales, carteleras y murales; (b) las rutinas para el trabajo en el aula que deben establecerse para las actividades académicas y las no académicas bien estructuradas y que resulten claras y coherentes; (c) el clima del aula comprende la atmósfera general de clase: amable u hostil, agradable o desagradable; en sí el clima es el resultado de actitudes del docente y los alumnos en la interacción recíproca en el aula; (d) reglas del aula: deben ser breves y especificas, con orientación positiva y comprenderse con claridad (Doyle, 1990) citado por Fried y Bursuck (1999); (e) empleo del tiempo libre: comprende dos momentos: (a) el empleo del tiempo de enseñanza que es la cantidad de tiempo dedicado a actividades académicas de forma significativa y provechosa; y (b) manejo del tiempo de transición, tiempo que se tarda de una actividad a otra, la misma debe contar con reglas especificas y un sistema de recompensas individualizado.

Los aspectos señalados por el autor, permiten comprender que es importante y necesario que el docente comprenda y asuma el proceso de integración con mejor información sobre el conocimiento del niño con necesidades especiales y por ende, de los aspectos básicos que comprende la organización del aula para asumir responsablemente la 
tarea de aplicar cada uno de dichos componentes de manera más adecuada para lograr el proceso de aprendizaje idóneo y armónico.

En este orden de ideas, Orellana (1991) explica que es en el salón de clase donde se localizan muchos esfuerzos y el maestro es quien tiene que hacer el registro de conductas, las cuales debe reforzar, modificar o erradicar; de esta forma, el maestro puede convertirse así en un terapeuta y a su vez funcionar como orientador de lo que deben hacer los padres y la familia toda, para prolongar en el seno de la comunidad, el trabajo en el aula.

En función a los aspectos discutidos anteriormente se puede destacar que en el salón de clase es donde se lleva un proceso dinámico, pues el docente organiza, planifica, observa, registra conductas de avances y comportamientos de los niños (as) para poder diagnosticar, evaluar, aplicar correctivos y metodologías; y ofrecer información a padres y representantes sobre el proceso de aprendizaje de los educandos que lo lleva a ejercer una gerencia adecuada en el aula, donde puede conjugar todos estos factores de fundamental importancia del proceso.

Desde este punto de vista, el niño (a) hiperactivo (a) en el contexto escolar, y en especial en el aula de clase, es visto (a) como indisciplinado (a), desobediente y despierta inquietud, preocupación y angustia tanto en los docentes como en los pares de su misma edad; esto es debido a los diversos comportamientos e incontroladas conductas y actitudes que asume este tipo de educando en su jornada escolar diaria. En el transcurso del tiempo y en diferentes ámbitos, el educando que presenta conductas hiperactivas ha sido estudiado y tratado de diversas maneras según el perfil de cada investigador o terapista, y los mismos coinciden en señalar que las conductas que los identifican son las mismas, tales como la impulsividad, la atención dispersa, la emotividad, las dificultades de aprendizaje, los problemas de comunicación y la interacción social.

En tal sentido, diferentes autores como Strauss y Kephart (1947), reseñado por Velett, (1981); Velasco (1980); Castro (1984); Uriarte (1989); Orellana (1991); Gargallo (1991), y Ávila (1992); Rief (1999); Joselevich, E. (2003); Arcos, T. y Fernandez, A. (2004); De La Garza, F. (2005); Asociación Americana de Psiquiatría (APA). DSM-IV-TR (2005); y Scandar, R: (2006) sostienen que el niño hiperactivo se caracteriza por la falta de atención, impulsividad y movimiento excesivo, siente necesidad de tocar y manipular todas las cosas, es desordenado y destructivo, tiene bajo concepto de sí mismo, y es impopular.
Estos estudiosos en el tema se concentran en señalar las conductas que presenta el niño hiperactivo como base para identificarlos y diagnosticarlos de manera clara y precisa en cualquier ambiente donde el niño hace vida activa. Asimismo, destacan que entre las características específicas que se evidencian en el aprendizaje se encuentran: coordinación motora pobre; presenta dificultad para dibujar, escribir, colorear, recortar; problemas de exactitud en las operaciones básicas, y lectura pobre reflejada en la dificultad para asociar sonidos alfabéticos y letras con fonemas (dislexia); también presentan problemas de articulación del habla (dislalia) y en la escritura tienen dificultad para reproducir las grafías (disgrafía).

Ante esta diversidad de conductas, cabe destacar la preocupación y el sentir de la madre antes de la llegada del niño (a) a la escuela, para indagar sobre los indicios de esta situación. Al respecto, acota Orellana (1991) que estos rasgos, actitudes, conductas, situaciones propias del niño hiperactivo, pueden ser resumidas muy bien en la siguiente forma: la madre informa cuando recuerda que el feto se movía mucho en el vientre, la irritabilidad expresada en el llanto, el sueño irregular e indica que cuando empieza a caminar se convierte en una tromba arrolladora.

Estas conductas presentes en el niño (a) desde la concepción hasta la llegada a la escuela reflejan la problemática que el niño hiperactivo presenta tanto en el proceso de aprendizaje como en la interrelación con el grupo. Según esto, se puede afirmar que los niños que presentan las características antes mencionadas, requieren atención no sólo a nivel de ellos en sí, sino a través de la familia, en la escuela, la comunidad, como lo refiere Castro (1984). Se evidencia frente a esta problemática, que los investigadores antes mencionados proponen un tratamiento integral, multidisciplinario, en donde el docente, de acuerdo con los padres y especialistas, colabore en la atención adecuada. Sin embargo, este tipo de tratamiento no se logra porque falta integrar esfuerzos entre especialistas y pedagogos para atender dicha problemática de manera integral donde se unifiquen criterios de los diferentes especialistas es decir, aplicar tratamiento pediátrico, psicológico, psicopedagógico y pedagógico de acuerdo a las necesidades del niño hiperactivo.

Indudablemente, hay que destacar que el docente de aula regular es quien tiene bajo su responsabilidad la atención pedagógica de un grupo de niños (as), entre los cuales existe un niño (a) con conductas hiperactivas. A este respecto, debe llevar un registro de observaciones de las conductas y compararlas con las anteriormente reseñadas para poder aseverar la presencia de un niño (a) hiperactivo (a) en el aula; para ello debe prepararse en relación a la temática expuesta para que pueda brindar una atención pedagógica adecuada a 
todo el grupo, comprender la problemática del niño (a) hiperactivo y dar orientaciones a la familia, para evitar que el niño hiperactivo al llegar a la escuela sea rechazado, descalificado y desescolarizado por su asidua indisciplina.

\section{APRENDIZAJE DEL NIÑO (A) HIPERACTIVO (A) EN EL AULA DE LA DIVERSIDAD}

El niño (a) hiperactivo (a) en el aula de la diversidad, requiere de un docente mediador del aprendizaje, conductor de procesos de socialización e interacción grupal y de formación de valores y hábitos necesarios para conformar su personalidad, lograr avances satisfactorios que le garanticen su permanencia dentro del sistema educativo. En cuanto a la enseñanza de habilidades, de organización y estudio, debe ser tarea del docente y los padres, abocarse a establecer normas y estrategias en la organización del estudio tanto en el aula como en su hogar. Rief (ob. cit.) presenta una serie de estrategias y habilidades para enseñar al niño con trastornos de atención e hiperactividad, dignos de tomar en cuenta en este estudio por su valor práctico, sencillo y orientador tanto para el maestro como para el resto de niños que son atendidos en el aula.

Otro elemento de observación en el niño con conductas hiperactivas y el resto de los niños que concurren al aula regular, es el proceso de lectura, escritura y cálculo denominado asignaturas instrumentales en donde el docente utiliza diversos métodos para hacer que el educando se apropie de este aprendizaje; pero en realidad estos procesos no se dan en todos los educandos de manera igual: unos avanzan progresivamente de manera acertada; pero otros, como el hiperactivo, se ve interferido por su intranquilidad y falta de atención para apropiarse en forma acabada del proceso. Esto se ve reflejado en los trabajos incompletos, borrones, cuadernos desordenados, sucios, inician la grafía de una palabra u oración y no la completan, todo esto producto de la atención dispersa, característica común de los niños con conductas hiperactivas.

Para entender el por qué de los desórdenes en el aprendizaje de la lectura, escritura y cálculo, es necesario comprender el proceso de aprendizaje de cuatro pasos: el primero, comprende el proceso de grabar la información en el cerebro; el segundo, consiste en organizarse y ser comprendido una vez grabada e interpretada la información, el tercero está referido a la memorización de la información. Finalmente, el cuarto, comprende comunicar la información desde el cerebro a las personas, o traducirse en acción sobre el ambiente. Cuando existe en el individuo una incapacidad para el aprendizaje, puede darse en alguno de estos cuatro pasos.
Por todo lo anterior, puede indicarse que el docente debe comprender al niño (a) con conductas hiperactivas, y en cuanto a las discapacidades para aprender sus preferencias perceptuales, para así determinar la manera de favorecer conocimientos, y ayudar a los niños (as) a adquirir aprendizajes significativos. También es responsabilidad del docente crear un clima de cooperación que coadyuve en el proceso de sociabilización de los niños (as). Para ello debe apoyarse en un aprendizaje cooperativo y facilitar el trabajo tanto del niño (a) con conductas hiperactivas, como al resto de educandos. Rief (ob. cit.), señala que el aprendizaje cooperativo es la estructura más beneficiosa y menos empleada en el aula, y presenta cinco elementos para su facilitación tales como: (a) la interdependencia positiva, (b) la interacción cara a cara, (c) la responsabilidad individual, (d) las habilidades interpersonales y de pequeños grupos, y (e) las reflexiones evolutivas del grupo.

En general, se puede afirmar que no basta con reunir a los niños (as) para lograr interdependencia positiva y deseos de cooperar; los alumnos deben ser organizados y estructurados para ejecutar sus actividades cooperativamente, de lo contrario, ellos ignoran al grupo y generan conductas impulsivas y disruptivas que interfieren en el proceso de enseñanza-aprendizaje y en el clima armónico de las relaciones interpersonales en el aula produciéndose un estado de intranquilidad y frustración en el docente, porque considera que el niño (a) no está obedeciendo órdenes, ni respetando normas preestablecidas, etiquetándolos entonces como desobedientes y mal educados.

Otros aspectos a considerar en el aprendizaje del niño hiperactivo son las dificultades de aprendizaje y el rendimiento escolar. A este respecto, Polaino y Ávila (2000), consideran que "El niño hiperactivo tiene dificultades en su desarrollo intelectual y perceptivo... y tiende a mostrar un desarrollo intelectual desigual, es decir, ocho años en capacidad de vocabulario acorde a la edad, pero la capacidad de comprender situaciones sociales es de cinco años” (p. 33-34).

Asimismo, dichos autores reseñan que las dificultades perceptivas son difíciles de definir por cuanto el niño no diferencia letras y sonidos similares y tiene poca capacidad para estructurar la información. También se pone de manifiesto la dificultad en la lectura y en la escritura donde se observa dificultad para escribir o dibujar y mala letra. En cálculo se olvidan “de llevar” en operaciones aritméticas básicas, saben sumar y restar con los dedos, pero no realizan operaciones mentales. En la lectura omiten palabras, silabas o renglones o no comprenden lo que leen. 
Estas aseveraciones evidencian la situación que presenta el niño (a) hiperactivo en el aula; cuando debe apropiarse del aprendizaje. Este alumno presenta falta de atención e impulsividad que intefiere en la adquisición de las habilidades y destrezas para leer, escribir y calcular. Esta situación encamina al docente a plantearse que además de ofrecerle una atención estandarizada, debe proporcionarle atención individualizada específica acorde con las necesidades e intereses del niño (a) hiperactivo (a), para ayudarlo así a apropiarse del aprendizaje y a relacionarse adecuadamente con el grupo.

\section{ROL DEL DOCENTE EN LA ATENCIÓN DEL NIÑO CON CONDUCTAS HIPERACTIVAS}

El docente es la persona clave dentro del proceso de aprendizaje, él es el responsable de la atención de un grupo de niños (as) que de acuerdo a la edad, cronológica y al nivel educativo pueden apropiarse eficientemente del aprendizaje. Asimismo, debe atender necesidades educativas especiales que requieren de su atención en el mismo grado y aula de clase. Al respecto, Ruiz (1992) acota que el docente tiene como misión optimizar el proceso de aprendizaje, aplicar estrategias, métodos, actuar objetivamente y establecer una comunicación clínica en el aula caracterizada por una relación terapéutica efectiva entre Docentes y Estudiantes.

Ante esta posición, el docente debe buscar la excelencia: planificando, organizando, dándole dirección y control al proceso de aprendizaje, propiciando una comunicación efectiva, tomando decisiones en cuanto al diseño, administrando estrategias pedagógicas pertinentes, observando las diferentes conductas expresadas por los participantes. Por tal motivo, el docente debe estar en constante búsqueda de su excelencia individual, vista ésta bajo la concepción de Much (1996) que la concebía como una forma de vida, una conducta, en la que a través de la práctica de virtudes tales como fortaleza, valentía, creatividad, esfuerzo y disciplina, se encuentra la felicidad en la calidad de vida y en el trabajo. Vista de esta manera, la calidad, en las organizaciones escolares ésta es el resultado de la excelencia en los individuos que la conforman. Lo planteado anteriormente representa un aspecto fundamental para todo docente que tenga la responsabilidad de aplicar un modelo pedagógico, orientado a la educación del niño (a) con déficit de atención con hiperactividad.

En cuanto a la atención del niño (a) con conductas hiperactivas en el aula regular, Velasco (1992) recomienda, en cuanto al tratamiento pedagógico, que al enseñar al niño se debe abordar el problema de la actitud y conducta que el docente no especializado adopta ante el comportamiento de los niños Hiperactivos que asisten a clase. De acuerdo a esta percepción, se comparte la idea de que es en el salón de clase donde se pueden observar en el niño (a) hiperactivo (a) tanto las conductas, como las necesidades de aprendizaje, aspectos éstos que el docente tiene que confrontar y saber manejar, para mantener la dinámica del aula y el progreso de este niño en las áreas académicas. En consecuencia, es el docente quien tiene a su disposición la observación del niño (a) hiperactivo en el contexto escolar, ya que es allí donde podrá interrelacionarse con el grupo de iguales y donde se le pueden brindar diversas experiencias pedagógicas que faciliten su aprendizaje, al demostrar sus habilidades, aptitudes y destrezas, y cumplir las normas del aula.

En atención a este planteamiento, Velasco (1992) reseña que es en el salón de clase donde el niño exterioriza más fácilmente los grandes grupos de síntomas: los que afectan la conducta del niño y los que producen las dificultades de aprendizaje. Por su parte, Uriarte (1989) destaca la importancia del maestro para entender la problemática, así como la persona clave en la terapéutica, ya que los maestros pueden proporcionar aquellas observaciones sobre las conductas que el niño hiperactivo exhibe en el aula, pues sólo en la escuela se le puede comparar con los demás compañeros. Esta acotación asigna al docente un rol fundamental en el diagnóstico y la atención del niño (a) hiperactivo en el aula regular.

En este orden de ideas, Mejía (1993) reseña que el docente que tiene bajo su responsabilidad compartir con niños hiperactivos en el aula, requiere de la orientación en aspectos como: disposición del docente, organización del ambiente escolar, confección y uso del material didáctico, reconocimiento de conductas, aplicación de técnicas para la atención adecuada, y, en la conducción de estrategias didácticas para inducir el proceso de aprendizaje. Así, la disposición del docente viene signada por el carácter, la personalidad y los valores que están íntimamente relacionados con su vocación profesional y ejercen influencia en la excelencia y ejecución de sus funciones. Es el carácter, como lo define Mejía (1999), el modo habitual de ser y comportarse de una persona que la hace ser ella misma, única y distinta a los demás. La personalidad, como lo reseña Stephen (1994) citando a Alport, es la suma de las formas en que una persona reacciona e interactúa con los demás.

De este modo, el pilar fundamental para encontrar el camino a la excelencia individual, es el carácter y la personalidad; ellos marcan diferencia entre los actos, conductas y comportamientos que cada individuo (educador) emite ante determinada situación de la vida personal y en el trabajo. Según esto, Mejía (ob. cit.) conceptualiza que la disposición 
del docente es la actitud que presenta ante el niño para ofrecerle un buen modelo de conducta a través de una adecuada atención, comunicación y comprensión empática. La organización del ambiente escolar, comprende la disposición que el docente le da a los recursos humanos y materiales para facilitar la ejecución de diversas estrategias de aprendizaje.

Para ello debe recibir la ayuda de un equipo multiprofesional, reconocer que el aula sirve de laboratorio y es allí donde puede observar reacciones, conductas de niños hiperactivos y el resto de los compañeros para poder llegar a hacer un inventario de sus conductas. Además, el docente debe entender su función de mediador ante la familia, para orientarla y reconocer que él se constituye en la figura fundamental para atender al niño (a) hiperactivo. Deberá diseñar programas que le permitan estimular sus habilidades y destrezas cognitivas, sociales y de aprendizaje y llevar registros, modificar el ambiente, entre otros.

El material didáctico, es el uso y la valoración que asigna el docente a los diversos materiales para trabajar con los niños, en especial el que presenta conductas hiperactivas en el aula de la diversidad. Además del material didáctico, en el ambiente escolar acorde para dar atención integral al niño, han de tenerse presente otros elementos como el espacio para recreo, el espacio para trabajo individual, el estado del mobiliario, los ruidos externos, la iluminación, la ventilación, la distribución de grupos, la comunicación y la autoestima que mediarán las relaciones niño-docente.

De igual manera, es indispensable que el docente esté capacitado y presto a observar las conductas del niño (a) hiperactivo ya que con éstas podrá hacer la valoración de aquellos comportamientos que se caracterizan por un nivel de actividad motora claramente mayor que el de los demás niños (as) de su misma edad, sexo, estatura y clase social. Además, el docente debe estar atento en el diseño de diversas estrategias didácticas que le faciliten la conducción de aprendizaje. Para Kindsvater, Wilen e Ishler, citados por Ruiz (1992): "la estrategia en el mundo educacional moderno, es lo que permite al docente guiar la toma de decisiones en cuanto al rol del docente, al rol del estudiante, selección de métodos y materiales instruccionales" (p. 134). Este autor condiciona el proceso de aprendizaje a la estrategia didáctica, como aspecto de vital importancia para que el docente y los estudiantes en el ejercicio de sus actividades, cumplan funciones, apliquen metodologías y utilicen materiales que faciliten el proceso de aprendizaje en concordancia con los avances tecnológicos y de acuerdo a los cambios que requiere en la actualidad.
En atención a la transformación del rol del docente, según Scandar, R. (2006) afirma que: “debe referirse a lograr comportamientos mínimos de atención, acomodar al niño en un sitio preferencial, mantener un contacto visual estrecho, eliminar distractores, mantener un nivel adecuado de estimulación, y mejorar las relaciones interpersonales en el grupo". (P. 87)

Considerando los retos que el docente afronta en el salón de clase para acondicionar un ambiente idóneo a las necesidades del niño, estas condiciones citadas por el autor en referencia, se consideran imprescindibles en el docente del aula regular y en especial a quien le corresponde atender e integrar niños (as) con necesidades especiales como es el caso de los niños hiperactivos (as).

En tal sentido, y reorientando la actitud del docente hacia la prevención y control de problemas de conducta que comúnmente presenta el niño hiperactivo en el aula de clase, Rief 1999, p. 39 reseña los factores más importantes en la conducción de estos, niños los cuales son: Claridad de las expectativas, enseñar lo que es aceptable e inaceptable en el aula, la estructura y la rutina, la predictividad, y consistencia, mucha práctica y modelado, revisión de expectativas y reglas conductuales, explicación de consecuencias claras y justas, ser persistente, comprensivo, flexible, paciente y pedir ayuda de especialistas.

El estudio de estos factores constituye un gran aporte para el docente ya que tanto el niño (a) con conductas hiperactivas como el resto de los niños (as) y hasta el mismo docente, necesitan sentirse queridos, seguros dentro de ciertas normas de convivencia y conocer qué se espera de ellos en cuanto al aprendizaje y su comportamiento. En general, el docente de aula regular en su acción pedagógica o quehacer permanente impartido en el aula, debe establecer una interacción recíproca con los educandos donde conjugue diversos elementos básicos tales como: distribución del ambiente escolar, elaboración y uso del material didáctico, estrategias didácticas dirigidas al proceso de lectura, escritura y cálculo, las relaciones interpersonales y la actitud del docente que permita llevar un proceso de aprendizaje óptimo e integral acorde con las necesidades e intereses de los niños y de acuerdo a la incorporación de diversos enfoques teóricos que le presenten las bases sólidas para la conducción adecuada, innovadora y creativa del niño con conductas hiperactivas en el aula regular. 


\section{ESTRATEGIAS PEDAGÓGICAS PARA LA ATENCIÓN DEL NIÑO (A) HIPERACTIVO}

El Docente dentro de este micromundo del aula, debe reflexionar y considerar que los seres humanos que atiende poseen grandes inquietudes y preocupaciones que se encuentran incapacitados para canalizar por sí mismos las dificultades que los agobian. Por esto el docente en su rutina diaria, ha de establecer sesiones de relajación, cuentos imaginarios guiados, moralejas, música, chistes, ejercicios, Así mismo, dramatizaciones por ejemplo para inducir al niño a liberarse y llegar a la serenidad. Rief (ob. cit.) recomienda estrategias para tranquilizarse y distenderse porque éstas proporcionan a los niños una sensación de paz y autocontrol.

Entre las estrategias que propone Rief (ob. cit.) se encuentran las siguientes: 1. La enseñanza multisensorial a través de estilos visuales, auditivos, táctiles, espaciales, verbales y conceptuales. 2. Las estrategias para el arte del lenguaje como las de prelectura y los organizadores gráficos, narración de cuentos, lectura oral. 3. Estrategias del lenguaje escrito tales como la enseñanza ortográfica, escritura manuscrita y calígrafa, organización de la hoja de papel, proceso de escritura y la enseñanza de puntuación. 4. Estrategias para la enseñanza de las matemáticas, uso de libros, hojas de ejercicios, programas de computación, evaluación y cuadernos de Matemática, enseñanza en equipo y representaciones gráficas. 5. Utilización de instrucciones y aprendizaje cooperativo que comprende la interdependencia positiva, la interacción cara-cara, las responsabilidades individuales, habilidades interpersonales y la evaluación del grupo.

Además de las Estrategias Pedagógicas señaladas anteriormente, Arco, J. y Fernandez, A. (2004), consideran para el manejo conductual en clase las siguientes técnicas destinadas al incremento de conductas deseadas: (a) reforzamiento positivo. Reforzar frecuentemente las conductas adecuadas en forma de atención, alabanzas, afecto, privilegios, entre otros; (b) programa de economía de ficha. Este tipo de programas consiste en la utilización de puntos o fichas que posteriormente se canjean por determinados reforzadores si el sujeto realiza la conducta deseada y finalmente retirada progresiva de la ficha para posibilitar la generalización; (c) contrato conductual. Es un procedimiento bastante útil que consiste en determinar conjuntamente con el niño la conducta que se desea que realice durante un tiempo determinado y las consecuencias derivadas de su cumplimiento o incumplimiento.

Finalmente según De la Garza, F. (2005), hace referencia a las estrategias que debe seguir el docente para dirigirse al niño hiperactivo: (a) tratar de mantener el contacto visual cuando se está hablando; (b) dar instrucciones claras concisas y diarias; (c) cuando las instrucciones son complejas y largas, tratar de simplificarlas o darlas por partes; (d) dar confianza al alumno para que pueda pedir ayuda; (e) si es necesario, revisar diariamente los libros de notas; (f) vigilar que lleve su tarea asignada escrita de manera que los padres puedan tomar conocimiento de ello.

\section{El equipo multiprofesional de apoyo al docente para la integración.}

Esbozar este tema obedece a la necesidad de apoyo que requiere el docente del Aula Integrada al aplicar programas de desarrollo individual para la atención del niño (a) con conductas hiperactivas en el aula regular. En la actualidad, la globalidad del niño (a), en especial, el que tiene problema para adaptarse a las exigencias del sistema educativo, requiere de un equipo multiprofesional, en donde el educando sea el centro de su propio proceso, que garantice en su acción la libertad, el amor, la credibilidad, capaz de dar una respuesta global y única, producto de la convergencia de conocimientos y aportes de cada una de las áreas de atención, tales como: la pedagógica, la médica, la psicológica y la social.

La multidisciplinaridad, tal como la define Alós, y Mardomingo (1991), comprende una pluralidad de especialistas y una funcionalidad interdisciplinaria que ofrece diversos servicios como: la prevención, detección, valoración, tratamiento y seguimiento. Los equipos multiprofesionales hacen más efectiva la integración del niño con conductas hiperactivas al Aula Regular Integrada; logran mayor eficacia en la elaboración e implementación de programas de atención individualizada, permiten el seguimiento, y pueden llegar a modificar el medio social.

\section{Integrantes del equipo multiprofesional}

Según Ponce 2001 , todo equipo multiprofesional debe estar constituido por diversos profesionales que se conforman en guías del docente y que debe atender conductas hiperactivas.

Un tratamiento eficaz aplicado a los niños afectados con TDA./H debe estar sustentado en un enfoque multimodal que integre profesionales de área de la salud y educación en un equipo multiprofesional conformado por: Médicos, Docentes, Psicopedagógos, Psicólogos Escolares, 
Terapeutas Ocupacional, los Terapeutas del Lenguaje y Trabajadores Sociales. (pàg,43),

Es necesario que el docente del Aula Regular Integrada, al tener en su grupo de educandos un niño(a) con conducta hiperactiva, debe estar dispuesto a ofrecerle atención integral, y para ello requiere de la ayuda y orientación de otros profesionales que le permitan realizar el proceso de integración escolar de manera adecuada de acuerdo a las necesidades e intereses de esta población. El docente debe entender que al formar parte de un equipo multiprofesional, él no es uno más de la colección de personas, y que no debe hacer su trabajo de manera anárquica, sin relacionar y compartir con el resto del grupo, si no que debe reflexionar, actuar y planificar correctamente al respecto, Alós y Mardomingo 1991, P. 26. El Docente debe tener un lenguaje común, un nivel de confianza, una relación de interdependencia, una comprobación de acercamiento a los fines permanentes y a los propósitos compartidos; establecer una escala de prioridades; El saber cómo ve cada uno en el equipo su prioridad, conocer la finalidad del modelo ( filosofía, objetivos, metas y prioridades), aceptar la filosofía adecuada, la estructura y la organización, analizar el modelo de comunicación utilizado entre especialistas y los padres; y evaluar resultados en relación a la cohesión y eficacia del funcionamiento.

De allí que es importante analizar y conjugar esfuerzos para que el docente comprenda, acepte y aplique los elementos señalados ya que éstos le permitirán constituirse en un miembro proactivo y receptivo, y le ayudará a tener una perspectiva de dónde va y a dónde quiere ir dentro del proceso de atención pedagógica del niño(a) con conductas hiperactivas en el Aula Regular Integrada. Cuando el docente no cuenta con la estructura de estos elementos, compartidos con un equipo multiprofesional, debe estar dispuesto a apropiarse de información, sobre los niños(as) con conductas hiperactivas a través de talleres, folletos, libros y de conocimientos, procesos y estrategias.

\section{Propósito del equipo multiprofesional en la integración.}

El Autor referido anteriormente, él mismo replantea como propósito de los equipos de apoyo, lo siguiente: -Servir de apoyo técnico especializado al docente de Educación Básica. -Ofrecer atención individualizada e integral al niño con conducta hiperactivas que asisten al aula regular. -Reorientar la función del docente ante el equipo multiprofesional, y la praxis pedagógica en la atención del niño hiperactivo en el aula regular. -Orientar la atención individualizada desarrollando en el niño los niveles de atención y concentración de acuerdo a las exigencias que requiere un determinado patrón de pedagogía.

\section{Funciones del Equipo Multiprofesional en la atención del niño hiperactivo}

El equipo multiprofesional cumple diversas funciones que el docente informado y preparado ha de conocer para poder insertarse en el mismo y así lograr la integración de funciones en la atención adecuada del niño(a) con conductas hiperactivas. Según Mardomingo (idem), el equipo multiprofesional debe ejercer sus funciones de acuerdo a 3 niveles: I Nivel: Prevención En este nivel el equipo debe dedicarse a modificar las condiciones sanitarias, sociales y educativas para prevenir dificultades de aprendizaje. II Nivel: Detección y Despistaje Los aspectos básicos que el equipo debe abordar son: detectar el problema lo antes posible, motivar la participación de los padres, mantener la estrecha colaboración entre los integrantes del equipo y establecer la relación con otros centros. III Nivel: Valoración En este momento el equipo unifica acuerdos, canaliza el problema y elabora programas de desarrollo oportunos e interviene observando y valorando. Estos niveles se pueden apreciar en el cuadro siguiente, donde se observan cada uno con su respectivas funciones.

\section{Cuadro 1}

Niveles y Funciones de los equipos de apoyo

\begin{tabular}{|c|c|}
\hline Niveles & Funciones \\
\hline Prevención & $\begin{array}{ll}\text { - } & \text { Detección de la familia de alto riesgo } \\
\text { - } & \text { Ubicar el problema en el marco escolar } \\
\text { - } & \text { Explorar actividades escolares } \\
\text { - } & \text { Localizar, y valorar necesidades e intereses del niño con conducta } \\
& \text { hiperactivas. } \\
\text { - } & \text { Adecuar el ámbito escolar y familiar a las necesidades del niño } \\
\text { hiperactivo. }\end{array}$ \\
\hline Valoración & $\begin{array}{ll}\text { - } & \text { Analizar datos de la historia del niño. } \\
\text { - } & \text { Explorar físicamente al Alumno. } \\
\text { - } & \text { Realizar pruebas formales e informales con base en la observación. } \\
\text { - } & \text { Levantar el inventario de conductas, y dificultades específicas de } \\
& \text { aprendizaje. } \\
\text { - } & \text { Valorar condiciones del aula y todo el ambiente escolar. } \\
\text { - } & \text { Estudiar socio-económicamente la familia. } \\
\text { - } & \text { Elaborar la síntesis multiprofesional única. }\end{array}$ \\
\hline Seguimiento & $\begin{array}{ll}\text { - } & \text { El equipo Interviene en la valoración de la síntesis multiprofesional. } \\
\text { - } & \text { Elaborar el programa de desarrollo individual. } \\
\text { - } & \text { Ubicar al sujeto en la institución o sistema acorde a sus } \\
\text { necesidades. } & \text { Apoyo al niño y a la familia y la ejecución de Programas Individualizados. }\end{array}$ \\
\hline
\end{tabular}


Las funciones antes mencionadas fueron contrastadas con la realidad existente en el ámbito escolar, a través de entrevistas a diversos especialistas que conforman equipos multiprofesionales de la institución de Educación Especial y del Ministerio de Salud. Los datos obtenidos demuestran que un equipo multiprofesional puede estar integrado por la Pediatra, un Psicólogo, un Psicopedagogo, un Trabajador Social y el docente de aula, y deben funcionar en los centros especiales y en las unidades de atención médica como: ambulatorios y hospitales.

Funciones del Pediatra: -Es el médico Especializado en Medicina Infantil. -Se encarga de las características físicas, biológicas y clínicas de la infancia. -Atiende las características diagnósticas, pronósticas y terapéuticas de la Clínica Pediátrica. -Defiende la adecuación de la vida del niño a su ambiente familiar, comunitario y sociedad en general; entre otras.

Funciones del Psicólogo: -Investiga y aplica los principios psicológicos con la finalidad de comprender la unicidad del cliente, y de ayudarle a funcionar significativa y eficientemente. -Comprende: a) la exploración, evaluación y diagnóstico de los problemas psicológicos del individuo utilizando los métodos de observación, la entrevista y la aplicación de diferentes tests. b) Una vez delimitado y especificado el problema se procede a la intervención psicológica mediante tratamientos y técnicas terapéuticas y de consejo a nivel individual y/o grupal.

El Psicólogo Escolar: Es un profesional que se encuentra integrado a un extenso grupo de personas formado por docentes, padres de familia, alumnos, entre otros. Las principales funciones del Psicólogo Escolar son: -Ayudar al alumno a la adaptación a la escuela. -Actuar técnicamente en el diagnóstico y exploración de los aspirantes a la escuela con el fin de determinar sus condiciones psicológicas y mentales para la actividad escolar. Realizar actividades de divulgación de los aspectos psicológicos que se refieren a la escuela o a la comunidad. -Orientar y asesorar sobre la toma de decisiones vocacionales, profesionales o escolares. -Asesorar a los profesores a cerca de la fatiga escolar en el estudiante, cambio de actitudes, sistema de participación en el aula, rendimiento escolar, entre otros.

Funciones del Psicopedagogo: -Atiende los factores psicológicos que participan en el proceso enseñanza - aprendizaje. -Aplica experiencias y principios psicológicos útiles y fundamentales para la investigación y el tratamiento de los problemas de la educación. -
Aplica principios psicológicos en los procesos del aprendizaje de las materias instrumentales.

Funciones del Trabajador Social: -Lleva un informe del ingreso del escolar. -Llena la anamnesis del escolar; la cual le permite obtener: a) datos personales; b) nivel de interacción en el medio familiar, escolar y social; c) información sobre la familia: componentes, nivel cultural, social y económico, salud, profesión, dinámica familiar, roles, relaciones; d) deficiencias que padece, aparición de síntomas de enfermedades, vacunaciones, evolución de los síntomas, trastornos del desarrollo, entre otros.

Docente Integrador: El docente integrador de niños hiperactivos en el aula regular debe tener presente los principios y recomendaciones, señalados por Gratch 2001, P. 27, mejorados y aplicados por el investigador para la atención de niños que presentan desorden por déficit de atención con hiperactividad: -Establecer y mantener el orden, la rutina, las normas de convivencia, los deberes, obligaciones, roles, organización y limpieza de pupitres, carpetas, cuadernos y lugar de trabajo. -Reforzar positivamente todas las veces que el alumno mantenga el orden y la organización. -El docente debe actuar con orden, organización y responsabilidad, ya que el niño aprende por imitación del modelaje del docente y adulto significante. -Fragmentar las tareas complejas en subtareas, a fin de aprovechar la capacidad de atención del niño al tiempo que los estimule a concluir lo ya comenzado. -El docente debe estimular al niño para que continúe y concluya los proyectos a mediano y largo plazo.

-En otro orden de ideas, debe tratar al niño con afecto, cariño, respeto y comprensión a fin de que se sienta apreciado, valorado y comprendido. -Motivar al niño para realizar tareas en las áreas de su mayor y mejor desempeño, de esta manera podrá reforzar su autoestima. Hacerle comprender que desde esas áreas de conocimiento o aptitudes puede contribuir con los demás de manera importante. -Alternar el trabajo que debe realizar en el pupitre con otras actividades que le permitan levantarse tales como: recoger material, repartir cuadernos. -Mantener contacto visual directo, utilizando gestos y expresiones faciales. -Llevar un registro de observación de las conductas de los niños donde se asienten sus aciertos, sus mejoras y progresos, y se destaque el valor del esfuerzo que realiza para superar su problema. De igual manera, se registrarán los cambios de conducta causados por las reacciones ante los fármacos. -Mantener tutoría con los padres cada quince días como mínimo y explicarles de forma clara y concreta durante dichas tutorías los problemas de conducta del niño y lo que ellos puedan hacer para controlarlos. -Tratar al niño de manera paciente, respetuosa y justa. Debe tomarse muy en cuenta el desempeño de 
acuerdo a sus capacidades y actitudes más que a sus deficiencias. "No lo avergüence, ni humille delante de sus compañeros”.

Los equipos multiprofesionales, en la actualidad están funcionando en los centros de educación especial, en hospitales tipo 2 y 3, pero no están atendiendo a los niño(a) con conductas hiperactivas como un área de la Educación Especial que debe conducirse de manera integral y coordinada entre el Ministerio de Salud Desarrollo Social, y el Ministerio de Educación, Cultura y Deporte; estos equipos deberían funcionar en cada centro dispensador de salud

Una vez creado el servicio y asignado los equipos, éstos deben realizar el estudio diagnóstico sobre la población de niños (as) con conductas hiperactivas existente en los diversos planteles educativos, incorporar a los docentes de aula regular que atiendan a éstos educándolos y brindar apoyo especializado tanto al educador como a la familia para atender dicha población dentro del ámbito escolar. De igual manera, deben realizarse visitas continuas al educador para orientar y dar seguimiento a los programas de atención individualizada.

\section{REFLEXIONES FINALES}

En conclusión, el docente de Aula Regular Integrada, como mediador del aprendizaje del niño (a) con conductas hiperactivas, debe atender sus función en el equipo multiprofesional tales como: llevar un registro de observación del niño hiperactivo, conocer los funciones de los especialistas, apropiarse de conocimientos teóricos y prácticos sobre la hiperactividad, elaborar el informe de referencia, aplicar algunas técnicas psicopedagógicas sencillas, establecer las redes de atención con la ayuda de los padres, mantener el orden, la organización y las normas en el aula, motivar al niño en su proceso de aprendizaje, aplicar las recomendaciones de los especialistas, y reunirse periódicamente con el equipo, permitir la ayuda de tutores, padres y adultos voluntarios en el trabajo con el niño(a) hiperactivo dentro del aula.

En el Aula Regular de la Primera Etapa de Educación Básica y los Primeros Años de La Educación Bolivariana se considera como elemento clave al Docente quién debe ejercer su acción pedagógica a través de una interacción recíproca con los educandos que le permita facilitar un proceso de aprendizaje integral a través de diversas Estrategias Pedagógicas aplicadas a la Atención del Niño Hiperactivo para así reforzar, dinamizar, afianzar y mediar el aprendizaje de manera organizada, planificada, correcta, específica e inclusiva.

\section{REFERENCIAS}

Alos J. Mardomingo M. (1991). Educación Especial. Editorial Cincel, S.A. Bogotá.

Arcos Tirado, J. y Fernández, A. (2004). Necesidades Educativas Especiales. Manual de Evaluación e Intervención Psicológica. Madrid - España: Editorial Mc. Graw Hill / Interamericana.

Asociación Americana de Psiquiatría (2005). Manual diagnóstico y Estadístico de los Trastornos Mentales (DSM-IV-TR). Barcelona - España: Edit. Masson.

Castro, P. (1984). El Niño Intranquilo. Ed. ULA. Mérida - Venezuela.

De la Garza Gutierrez, F. (2005). Hiperactividad y Déficit de Atención en Niños y Adultos. México: Editorial Trilla.

Fried y Bursuck (1999). Alumnos con dificultades. Argentina: Edit. Troquel.

Gargallo, B. (1991). Hijos hiperactivos. Perú: Edic. CEAC.

Goleman (1996). La inteligencia emocional. Buenos Aires: Edit. Javier Vergara.

Gratch, L, (2001) El Trastorno por Déficit de Atención. Buenos Aires: Editorial Panamericana

Heller (1996). El Arte de Enseñar con todo el Cerebro. Caracas - Venezuela

Mejía, M. (1993). Orientación al docente de la Primera Etapa de Educación Básica para la atención del niño hiperactivo en el aula. Tesis de grado.

Mejía, M. (1999). Excelencia individual. Clave de I excelencia en las organizaciones. Material mimeografiado. Barquisimeto, Venezuela.

Ministerio de Educación (1996). Resolución N 2005. Caracas, Diciembre 2, 1996. 
Much (1996). Más allá de la excelencia y de la calidad total. México: Ed. Trillas.

Orellana, J. (1991). Inatención e intranquilidad. Caracas: Edic. Nuevo Enfoque.

Polaina y Avila (2000). Cómo vivir con el niño hiperactivo(a). Madrid, España: Edic. Narcea.

Ponce, S. (2001). Bases Neurológicas del Niño Inquieto. Venezuela: Editorial Litovenca.

Rief, S. (1999). Cómo tratar y Enseñar al Niño con Problemas de Atención e Hiperactividad. Buenos Aires: Editorial Paidos.

Ruiz, L. (1992). Gerencia en el aula. Nirgua: Instivoc.

Scandar, R. (2006). Inquietos, Distraídos, ¿Diferentes?. Buenos Aires: Editorial EDIBA. SRL.

Uriarte (1989). Hiperquinecia. México: Edit. Trillas.

Valett, R. (1981). Niños hiperactivos. Guía para la familia y la escuela. España: Edit. Cincel.

Velasco, R. (1992). El niño hiperactivo. México: Trillas. 